\title{
Polynomial Equivalence Problems: Algorithmic and Theoretical Aspects
}

\author{
Jean-Charles Faugère ${ }^{1}$ and Ludovic Perret ${ }^{2}$ \\ ${ }^{1}$ LIP6, 8 rue du Capitaine Scott, F-75015, France \\ Jean-Charles .Faugere@lip6.fr \\ ${ }^{2}$ UCL, Crypto Group, Microelectronic Laboratory, Place du Levant, \\ 3 Louvain-la-Neuve, B 1348, Belgium \\ ludovic.perret@uclouvain. be
}

\begin{abstract}
The Isomorphism of Polynomials (IP) [28], which is the main concern of this paper, originally corresponds to the problem of recovering the secret key of a $\mathrm{C}^{*}$ scheme [26]. Besides, the security of various other schemes (signature, authentication [28], traitor tracing [5], ...) also depends on the practical hardness of IP. Due to its numerous applications, the Isomorphism of Polynomials is thus one of the most fundamental problems in multivariate cryptography. In this paper, we address two complementary aspects of IP, namely its theoretical and practical difficulty. We present an upper bound on the theoretical complexity of "IP-like" problems, i.e. a problem consisting in recovering a particular transformation between two sets of multivariate polynomials. We prove that these problems are not NP-Hard (provided that the polynomial hierarchy does not collapse). Concerning the practical aspect, we present a new algorithm for solving IP. In a nutshell, the idea is to generate a suitable algebraic system of equations whose zeroes correspond to a solution of IP. From a practical point of view, we employed a fast Gröbner basis algorithm, namely $\mathrm{F}_{5}$ [17], for solving this system. This approach is efficient in practice and obliges to modify the current security criteria for IP. We have indeed broken several challenges proposed in literature $[28,29,5]$. For instance, we solved a challenge proposed by O. Billet and H. Gilbert at Asiacrypt'03 [5] in less than one second.
\end{abstract}

Keywords: Public-Key Cryptography, Cryptanalysis, Isomorphism of Polynomials (IP), Gröbner bases, $F_{5}$ algorithm.

\section{Introduction}

Multivariate cryptography - which can be roughly defined as the cryptography using polynomials in several variables - offers a relatively wide spectrum of problems that can be used in public-key cryptography. The Isomorphism of Polynomials (IP) lies in this family [28]. Briefly, this problem consists in recovering a particular transformation between two sets of multivariate polynomials permitting to obtain one set from the other. It originally corresponds to the problem of recovering the secret key of a $\mathrm{C}^{*}$ scheme [26]. Besides, the security of

The original version of this chapter was revised: The copyright line was incorrect. This has been corrected. The Erratum to this chapter is available at DOI: 10.1007/978-3-540-34547-3_36

S. Vaudenay (Ed.): EUROCRYPT 2006, LNCS 4004, pp. 30-47, 2006.

(C) Springer-Verlag Berlin Heidelberg 2006 
several other schemes is directly based on the practical difficulty of IP, namely the authentication/signature schemes proposed by J. Patarin at Eurocrypt'96 [28], and the traitor tracing scheme described by O. Billet and H. Gilbert at Asiacrypt'03 [5]. We also mention that IP is in a certain manner related to the security of Sflash [13] - the signature scheme recommended by the European consortium Nessie for low-cost smart cards [27] - and can be alternatively viewed as the problem of detecting affine equivalence between S-Boxes [6]. All in all, one can consider the hardness of IP as one of the major issues in multivariate cryptography. The goal of this paper is to provide new insights on the theoretical and practical complexity of IP and some of its relevant variants.

\subsection{Previous Work}

To the best of our knowledge, the most significant results concerning IP are presented in [11], where an upper bound on the theoretical complexity of IP is given. Nevertheless, we point out that the proof provided is actually not complete. Anyway, the upper bound presented in that paper is original and general. It is indeed based on a group theoretic approach of IP and actually dedicated to "IP-like" problems. A new algorithm for solving IP, called "To and Fro", is also described in [11]. This algorithm is however devoted to special instances of IP, namely the ones corresponding to a public key of $\mathrm{C}^{*}[26]$. Thus, it can not be used for solving generic instances of IP. This is not the case for the algorithm presented here. Besides, we present in Section 4 experimental results demonstrating that our algorithm outperforms the "To and Fro" method. Finally, we would like to mention a result due to W. Geiselmann, R. Steinwandt, and T. Beth [23]. In the context of $\mathrm{C}^{*}$, they showed how to easily recover the affine parts of a solution of IP. A similar property also holds in the context of HFE [20]. Such a kind of result does not exist for generic instances of IP. Nevertheless, it means that in the cryptographic context we can focus our attention on the linear variant of IP, called 2PLE here.

\subsection{Organization of the Paper and Main Results}

The paper is organized as follows. We begin in Section 2 by introducing our notation and defining essential tools of our algorithm, namely varieties and Gröbner bases. A recent algorithm (i.e. $\mathrm{F}_{5}[17]$ ) for computing these bases is also succinctly described. Finally, we define more formally the Isomorphism of Polynomials (IP) and two of its variants, namely the Isomorphism of Polynomials with one Secret (IP1S) [28], and the linear variant of IP that we name 2PLE. In Section 3, we show that these problems are actually particular instances of a more general problem that we call Polynomial Equivalence (PE). This problem provides a formal definition of an "IP-like" problem. Using classical results of group theory, we conclude this section by providing an upper bound on the theoretical hardness of PE. A new algorithm for solving 2PLE is presented in Section 4. The idea is to generate a suitable polynomial system of equations whose zeroes correspond to a solution of IP. In order to construct this system, we also provide some specific properties of 2 PLE. From a practical point of view, we used the 
most recent (and efficient) Gröbner basis algorithm, namely $\mathrm{F}_{5}$ [17], for solving this system. It is difficult to obtain a complexity bound really reflecting the practical behavior of the $\mathrm{F}_{5}$ algorithm. We therefore carried out experimental results illustrating the practical efficiency of our approach. We have indeed broken several challenges proposed in literature $[28,29,5]$. For instance, we solved a challenge proposed by O. Billet and H. Gilbert at Asiacrypt'03 [5] in less than one second.

\section{Preliminaries}

The notation used throughout this paper is the following. We denote by $\mathbb{F}_{q}$ the finite field with $q=p^{r}$ elements ( $p$ a prime, and $r \geq 1$ ), and by $\mathcal{M}_{n, u}\left(\mathbb{F}_{q}\right)$ the set of $n \times u$ matrices whose components are in $\mathbb{F}_{q}$. As usual, $G L_{n}\left(\mathbb{F}_{q}\right)$ represents the set of invertible matrices of $\mathcal{M}_{n, n}\left(\mathbb{F}_{q}\right)$, and $A G L_{n}\left(\mathbb{F}_{q}\right)$ denotes the cartesian product $G L_{n}\left(\mathbb{F}_{q}\right) \times \mathbb{F}_{q}^{n}$. Finally, let $\mathbf{x}=\left(x_{1}, \ldots, x_{n}\right)$, and $\mathbb{F}_{q}[\mathbf{x}]=\mathbb{F}_{q}\left[x_{1}, \ldots, x_{n}\right]$, be the polynomial ring in the $n$ indeterminates $x_{1}, \ldots, x_{n}$ over $\mathbb{F}_{q}$. By convention, a boldfaced letter will always refer to a row vector.

\subsection{Gröbner Bases}

We define now two essential notions of this paper, namely varieties and Gröbner bases. For a more thorough introduction to these tools, we refer to $[1,15]$.

Let $\mathbf{p}=\left(p_{1}, \ldots, p_{s}\right)$ be polynomials in $\mathbb{F}_{q}[\mathbf{x}]$. We shall call $\mathcal{I}=\left\langle p_{1}, \ldots, p_{s}\right\rangle=$ $\left\{\sum_{k=1}^{s} p_{k} u_{k}, u_{1}, \ldots, u_{k} \in \mathbb{F}_{q}[\mathbf{x}]\right\} \subset \mathbb{F}_{q}[\mathbf{x}]$ the ideal generated by $p_{1}, \ldots, p_{s}$, and denote by $V(\mathcal{I})=\left\{\mathbf{z} \in \mathbb{F}_{q}^{n}: p_{i}(\mathbf{z})=0, \forall i, 1 \leq i \leq s\right\}$ the variety associated to $\mathcal{I}$. Gröbner bases provide a method for computing this variety. Informally, a Gröbner basis of an ideal $\mathcal{I}$ is a computable generator set of $\mathcal{I}$ with "good" algorithmic properties. These bases are defined with respect to monomial orders. Here, we will use the lexicographical (LEX) and degree reverse lexicographical (DRL) orders, which are defined as follows:

Definition 1. Let $\alpha=\left(\alpha_{1}, \ldots, \alpha_{n}\right)$ and $\beta=\left(\beta_{1}, \ldots, \beta_{n}\right) \in \mathbb{N}^{n}$. Then: $-x_{1}^{\alpha_{1}} \cdots x_{n}^{\alpha_{n}} \prec_{L E X} x_{1}^{\beta_{1}} \cdots x_{n}^{\beta_{n}}$, if the left-most nonzero entry of $\alpha-\beta$ is positive. - $x_{1}^{\alpha_{1}} \cdots x_{n}^{\alpha_{n}} \prec_{D R L} x_{1}^{\beta_{1}} \cdots x_{n}^{\beta_{n}}$, if $\sum_{i=1}^{n} \alpha_{i}>\sum_{i=1}^{n} \beta_{i}$, or $\sum_{i=1}^{n} \alpha_{i}=\sum_{i=1}^{n} \beta_{i}$ and the right-most nonzero entry of $\alpha-\beta$ is negative.

To define Gröbner bases, we need to introduce the following definitions.

Definition 2. For any $n$-tuple $\alpha=\left(\alpha_{1}, \ldots, \alpha_{n}\right) \in \mathbb{N}^{n}$, we denote by $\mathbf{x}^{\alpha}$ the monomial $x_{1}^{\alpha_{1}} \cdots x_{n}^{\alpha_{n}}$. We shall define the total degree of this monomial by the sum $\sum_{i=1}^{n} \alpha_{i}$. The leading monomial of a polynomial $p \in \mathbb{F}_{q}[\mathbf{x}]$ is the largest monomial (w.r.t some monomial ordering $\prec$ ) among the monomials of $p$. This leading monomial will be denoted by $\operatorname{LM}(p, \prec)$. The degree of $p$, denoted deg $(p)$, is the total degree of $\operatorname{LM}(p, \prec)$. Finally, the maximal total degree of $p$ is the maximal total degree of the monomials occurring in $p$.

We are now in a position to define one of the main objects of this paper. 
Definition 3. A set of polynomials $G$ is a Gröbner basis - w.r.t. a monomial ordering $\prec-$ of an ideal $\mathcal{I}$ in $\mathbb{F}_{q}[\mathbf{x}]$ if, for all $p \in \mathcal{I}$, there exists $g \in G$ such that $\operatorname{LM}(g, \prec)$ divides $\operatorname{LM}(p, \prec)$.

Gröbner bases are a fundamental tool to study algebraic systems in theory and practice. They provide an algorithmic solution to several problems related to polynomial systems (see [1] for instance). We pay here particular attention to Gröbner bases computed for a lexicographical ordering. It offers a way of simplifying an algebraic system by giving an equivalent system with a structured shape. A lexicographical Gröbner basis of a zero-dimensional system (i.e. with a finite number of zeroes over the algebraic closure) is indeed always as follows:

$$
\left\{f_{1}\left(x_{1}\right)=0, f_{2}\left(x_{1}, x_{2}\right)=0, \ldots, f_{k_{2}}\left(x_{1}, x_{2}\right)=0, f_{k_{2}+1}\left(x_{1}, x_{2}, x_{3}\right)=0, \ldots, \ldots\right\}
$$

To compute the variety, we simply have to successively eliminate variables by computing zeroes of univariate polynomials and back-substituting results. However, computing a Gröbner basis w.r.t. a lexicographical order is in practice much slower than computing a Gröbner basis w.r.t. another monomial ordering. It is usually for a DRL order that the computation of Gröbner bases is the fastest in practice. Algorithms changing the monomial ordering of a Gröbner basis permit to handle efficiently this problem. The FLGM algorithm [19] allows to transform a Gröbner basis w.r.t. some monomial ordering into a lexicographical Gröbner basis in the zero-dimensional case and is polynomial-time.

The historical method for computing Gröbner bases is Buchberger's algorithm $[9,8]$. Recently, more efficient algorithms have been proposed. The $\mathrm{F}_{4}$ algorithm [16] is based on the intensive use of linear algebra methods. In short, the arbitrary choices - which limit the practical efficiency of Buchberger's algorithm - are replaced by computational strategies related to classical linear algebra problems (mainly the computation of a row echelon form).

In [17], a new criterion (the $\mathrm{F}_{5}$ criterion) for detecting useless computations has been proposed. We mention that Buchberger's algorithm spends $90 \%$ of its time to perform these useless computations. Under some regularity conditions, it has been proved that all useless computations can be avoided. A new algorithm, called $\mathrm{F}_{5}$, has then been built using this criterion and linear algebra methods. Briefly, it constructs incrementally the following matrices in degree $d$ :

$$
\begin{array}{r}
m_{1} \succ m_{2} \succ m_{3} \ldots \\
t_{d} f_{1} \\
t_{2} f_{2} \\
t_{3} f_{3} \\
\ldots
\end{array}\left[\begin{array}{lllll}
\ldots & \ldots & \ldots & \ldots \\
\ldots & \ldots & \ldots & \ldots & \ldots \\
\ldots & \ldots & \ldots & \ldots
\end{array}\right]
$$

where the indices of the columns are monomials sorted for the admissible ordering $\prec$ and the rows are product of some polynomials $f_{i}$ by some monomials $t_{j}$ such that $\operatorname{deg}\left(t_{j} f_{i}\right) \leq d$. For a regular system ([17]) the matrices $A_{d}$ are of full rank. In a second step, row echelon forms of theses matrices are computed, i.e. 


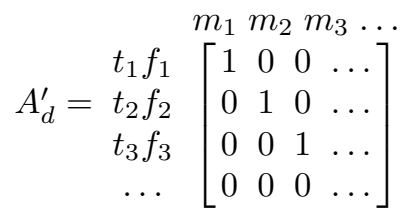

For $d$ sufficiently large, $A_{d}^{\prime}$ contains a Gröbner basis of the ideal considered. Important parameters to evaluate the complexity of $\mathrm{F}_{5}$ is the maximal degree $d$ occurring in the computation and the size of the matrix $A_{d}$. The overall cost is thus dominated by $\left(\# A_{d}\right)^{3}$. Very roughly, $\left(\# A_{d}\right)$ can be approximated by $O\left(n^{d}\right)$. A more precise complexity analysis can be found in $[3,4]$.

From a practical point of view, the gap with other algorithms computing Gröbner basis is consequent. To date, $\mathrm{F}_{5}$ is the most efficient method for computing Gröbner bases, and hence zero-dimensional varieties. In particular, it has been proved [2] - from both a theoretical and practical point of view - that XL [14] is less efficient than $\mathrm{F}_{5}$. Due to the range of examples that become computable with $\mathrm{F}_{5}$, Gröbner basis can be considered as a reasonable computable object in real scale applications. For systems arising in cryptography, $\mathrm{F}_{5}$ has for instance given impressing results on HFE [18].

\subsection{Isomorphism of Polynomials and Related Problems}

Before defining formally IP, we briefly come back here to the origin of this problem. To do so, we describe the encryption scheme called $\mathrm{C}^{*}[26]$. The public key of this system is a set of multivariate quadratic polynomials $\mathbf{b}=$ $\left(b_{1}(\mathbf{x}), \ldots, b_{n}(\mathbf{x})\right) \in \mathbb{F}_{q}[\mathbf{x}]^{n}$. These polynomials are obtained by applying two bijective affine transformations $(S, \mathbf{V})$ and $(U, \mathbf{V})$ of $A G L_{n}\left(\mathbb{F}_{q}\right)$ to a particular set of polynomials $\mathbf{a}=\left(a_{1}(\mathbf{x}), \ldots, a_{n}(\mathbf{x})\right) \in \mathbb{F}_{q}[\mathbf{x}]^{n}$. That is:

$$
\left(b_{1}(\mathbf{x}), \ldots, b_{n}(\mathbf{x})\right)=\left(a_{1}(\mathbf{x} S+\mathbf{T}), \ldots, a_{n}(\mathbf{x} S+\mathbf{T})\right) U+\mathbf{V},
$$

denoted $\mathbf{b}(\mathbf{x})=\mathbf{a}(\mathbf{x} S+\mathbf{T}) U+\mathbf{V}$ in the sequel.

To encrypt, we simply evaluate a message $\mathbf{m} \in \mathbb{F}_{q}^{n}$ on $\mathbf{b}$, i.e. $\left(b_{1}(\mathbf{m}), \ldots, b_{n}(\mathbf{m})\right)$. To recover the correct plaintext, the legitimate recipient uses the bijectivity of the affine transformations combined with the particular structure of the polynomials of a. How these polynomials are constructed is not relevant here. But, due to particular constraints, the polynomials of $\mathbf{a}$ are always considered as a public data. The secret key of $\mathrm{C}^{*}$ is constituted of $(S, \mathbf{T}),(U, \mathbf{V}) \in A G L_{n}\left(\mathbb{F}_{q}\right)$.

The first approach for attacking this scheme consists in trying to retrieve the message corresponding to a ciphertext $\mathbf{c} \in \mathbb{F}_{q}^{n}$, i.e. finding a zero of $\mathbf{b}(\mathbf{x})=\mathbf{c}$. This corresponds to solving a particular instance of the so-called MQ problem, which is NP-Hard in general $[10,22]$. We emphasize that such a kind of result uniquely guarantees the worst-case hardness and does not provide any information concerning the average-case difficulty. For instance, J.-C. Faugère and A. Joux proposed a polynomial-time algorithm for solving instances of MQ corresponding to the public key of HFE [18], which is an extension of $\mathrm{C}^{*}$.

Another approach for breaking $\mathrm{C}^{*}$ consists in attempting to recover the affine transformations hiding the structure of $\underline{a}$. That is, extracting the secret key from 
the public key. This problem, introduced by J. Patarin at Eurocrypt'96 [28], is defined as follows:

\section{Isomorphism of Polynomials (IP)}

Input: $\mathbf{a}=\left(a_{1}, \ldots, a_{u}\right)$, and $\mathbf{b}=\left(b_{1}, \ldots, b_{u}\right)$ in $\mathbb{F}_{q}[\mathbf{x}]^{u}$.

Question: Find - if any - $(S, \mathbf{V}) \in A G L_{n}\left(\mathbb{F}_{q}\right)$ and $(U, \mathbf{V}) \in A G L_{u}\left(\mathbb{F}_{q}\right)$, s. t.:

$$
\mathbf{b}(\mathbf{x})=\mathbf{a}(\mathbf{x} S+\mathbf{V}) U+\mathbf{V}
$$

More precisely, it is usually the linear variant of IP which is considered in practice $[28,5]$. That is, when the vectors $\mathbf{T}$ and $\mathbf{V}$ are both equal to the null vector. This problem, that we call $2 \mathrm{PLE}$ is the following:

Input: $\mathbf{a}=\left(a_{1}, \ldots, a_{u}\right)$, and $\mathbf{b}=\left(b_{1}, \ldots, b_{u}\right)$ in $\mathbb{F}_{q}[\mathbf{x}]^{u}$.

Question: Find - if any - $(S, U) \in G L_{n}\left(\mathbb{F}_{q}\right) \times G L_{u}\left(\mathbb{F}_{q}\right)$, such that:

$$
\mathbf{b}(\mathbf{x})=\mathbf{a}(\mathbf{x} S) U .
$$

However, it is without solving any of the two problems mentioned above that J. Patarin proposed a full cryptanalysis of $\mathrm{C}^{*}[30]$. This attack uses the very particular structure of the polynomials of $\underline{a}$. This result thus does not then affect at all the practical hardness of IP. The security estimate provided for this problem [29] is based on the complexity of the "To and Fro" (TF) algorithm $[11,12]$, which is $q^{n / 2}$ for quadratic polynomials, and $q^{n}$ otherwise.

In the rest of this paper, $\left(\mathbf{a}=\left(a_{1}, \ldots, a_{u}\right), \mathbf{b}=\left(b_{1}, \ldots, b_{u}\right)\right)$ will always denote an element of $\mathbb{F}_{q}[\mathbf{x}]^{u} \times \mathbb{F}_{q}[\mathbf{x}]^{u}$. We will always suppose that all the polynomials of a have the same maximal total degree noted $D$ (in the practical applications, we have $2 \leq D \leq 4)$. Note that, if $\mathbf{b}(\mathbf{x})=\mathbf{a}(\mathbf{x} S) U$, for some $(S, U) \in G L_{n}\left(\mathbb{F}_{q}\right) \times$ $G L_{u}\left(\mathbb{F}_{q}\right)$, then the polynomials of $\mathbf{b}$ must have the same maximal total degree than the ones of a, i.e. $D$.

\section{A Unified Point of View}

The Isomorphism of Polynomials and 2PLE problems have actually a very similar formulation. An input of these problems is formed of two systems of multivariate polynomials and the question consists in recovering a particular transformation permitting to express one system in function of the other. All transformations have the same characteristic: inducing a group action on $\mathbb{F}_{q}[\mathbf{x}]^{u}$. Recall that a group $(G, \cdot)$, with identity element $e$, acts on $\mathbb{F}_{q}[\mathbf{x}]^{u}$ if there exists a map $\phi: G \times \mathbb{F}_{q}[\mathbf{x}]^{u} \rightarrow \mathbb{F}_{q}[\mathbf{x}]^{u}$ such that $\phi(e, \mathbf{p})=\mathbf{p}$, for all $\mathbf{p} \in \mathbb{F}_{q}[\mathbf{x}]^{u}$, and:

$$
\phi\left(g, \phi\left(g^{\prime}, \mathbf{p}\right)\right)=\phi\left(g \cdot g^{\prime}, \mathbf{p}\right) \text {, for all } g, g^{\prime} \in G \text {, and for all } \mathbf{p} \in \mathbb{F}_{q}[\mathbf{x}]^{u} .
$$

Remark 1. In order to simplify the notations, we will write $G$ instead of $(G, \cdot)$.

For $2 \mathrm{PLE}$, one can then easily check that $G L_{n}\left(\mathbb{F}_{q}\right) \times G L_{u}\left(\mathbb{F}_{q}\right)$ acts on $\mathbb{F}_{q}[\mathbf{x}]^{u}$ through:

$$
\begin{aligned}
\phi_{2 \mathrm{PLE}}: G L_{n}\left(\mathbb{F}_{q}\right) \times G L_{u}\left(\mathbb{F}_{q}\right) \times \mathbb{F}_{q}[\mathbf{x}]^{u} & \rightarrow \mathbb{F}_{q}[\mathbf{x}]^{u} \\
((S, U), \mathbf{a}) & \mapsto \mathbf{a}(\mathbf{x} S) U
\end{aligned}
$$


Similarly for IP, $A G L_{n}\left(\mathbb{F}_{q}\right) \times A G L_{u}\left(\mathbb{F}_{q}\right)$ acts on $\mathbb{F}_{q}[\mathbf{x}]^{u}$ through:

$$
\begin{aligned}
\phi_{\mathrm{IP}}: A G L_{n}\left(\mathbb{F}_{q}\right) \times A G L_{u}\left(\mathbb{F}_{q}\right) \times \mathbb{F}_{q}[\mathbf{x}]^{u} & \rightarrow \mathbb{F}_{q}[\mathbf{x}]^{u} \\
((S, \mathbf{T}),(U, \mathbf{V}), \mathbf{a}) & \mapsto \mathbf{a}(\mathbf{x} S+\mathbf{T}) U+\mathbf{V}
\end{aligned}
$$

This observation naturally leads to the introduction of the following problem. Let $(G, \cdot)$ be a group, and $\phi: G \times \mathbb{F}_{q}[\mathbf{x}]^{u} \rightarrow \mathbb{F}_{q}[\mathbf{x}]^{u}$ be an action of $G$ on $\mathbb{F}_{q}[\mathbf{x}]^{u}$. Given $(\mathbf{a}, \mathbf{b}) \in \mathbb{F}_{q}[\mathbf{x}]^{u} \times \mathbb{F}_{q}[\mathbf{x}]^{u}$, the problem we call Polynomial Equivalence, with respect to $(G, \cdot)$ and $\phi$ - and denoted by $\operatorname{PE}(G, \phi)$ - is the one of finding (if any) $g \in G$, verifying:

$$
\mathbf{b}=\phi(g, \mathbf{a})
$$

denoted $\mathbf{a} \equiv_{(G, \phi)} \mathbf{b}$ in the sequel. This formulation is very convenient since it procures a unified description of IP and 2PLE. Indeed, $\operatorname{PE}\left(G L_{n}\left(\mathbb{F}_{q}\right) \times G L_{u}\left(\mathbb{F}_{q}\right)\right.$, $\left.\phi_{2 \mathrm{PLE}}\right)=2 \mathrm{PLE}$, and $\mathrm{PE}\left(A G L_{n}\left(\mathbb{F}_{q}\right) \times A G L_{u}\left(\mathbb{F}_{q}\right), \phi_{\mathrm{IP}}\right)=\mathrm{IP}$. More generally, PE provides a unified description of "IP-like" problems. In our mind, such a kind of problems consists in recovering a particular transformation between two sets of multivariate polynomials. For instance, the Isomorphism of Polynomials with one Secret (IP1S) - introduced at Eurocrypt'96 by J. Patarin [28] - falls into this new formalism. This problem, which can be used to design an authentication (resp. signature) scheme [28], is as follows. Given $(\mathbf{a}, \mathbf{b}) \in \mathbb{F}_{q}[\mathbf{x}]^{u} \times \mathbb{F}_{q}[\mathbf{x}]^{u}$, find - if any - $(S, \mathbf{T}) \in A G L_{n}\left(\mathbb{F}_{q}\right)$, such that $\mathbf{b}(\mathbf{x})=\mathbf{a}(\mathbf{x} S+\mathbf{T})$. Using our formalism, we immediately obtain that $\operatorname{PE}\left(A G L_{n}\left(\mathbb{F}_{q}\right), \phi_{\text {IP1S }}\right)=$ IP1S, with $\phi_{\text {IP1S }}: A G L_{n}\left(\mathbb{F}_{q}\right) \times \mathbb{F}_{q}[\mathbf{x}]^{u} \rightarrow \mathbb{F}_{q}[\mathbf{x}]^{u},((S, \mathbf{T}), \mathbf{a}(\mathbf{x})) \mapsto \mathbf{a}(\mathbf{x} S+\mathbf{T})$. Finally, the following lemma justifies the use of the word equivalence in $\mathrm{PE}$.

Lemma 1. Let $(G, \cdot)$ be a group, and $\phi: G \times \mathbb{F}_{q}[\mathbf{x}]^{u} \rightarrow \mathbb{F}_{q}[\mathbf{x}]^{u}$ be an action of $G$ on $\mathbb{F}_{q}[\mathbf{x}]^{u}$. Then, $\equiv_{(G, \phi)}$ is an equivalence relation on $\mathbb{F}_{q}[\mathbf{x}]^{u}$.

\subsection{Polynomial Equivalence Problems and Group theory}

In the Graph Isomorphism context, the introduction of group theory concepts permitted to achieve significant advances from both a theoretical and algorithmic point of view $[24,21]$. The formalism previously given permits to naturally extend these results to Polynomial Equivalence problems.

Definition 4. Let $(G, \cdot)$ be a group. We shall call Aut $(G, \phi)(\mathbf{a})=\{g \in G$ : $\phi(g, \mathbf{a})=\mathbf{a}\}, \operatorname{Aut}_{(G, \phi)}(\mathbf{b})=\{g \in G: \phi(g, \mathbf{b})=\mathbf{b}\}$, the automorphism groups of $\mathbf{a}$ and b w.r.t. $(G, \phi)$. We shall also set $S_{(G, \phi)}(\mathbf{a}, \mathbf{b})=\{g \in G: \mathbf{b}=\phi(g, \mathbf{a})\}$.

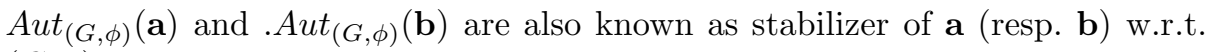
$(G, \phi)$. However, we will rather call these sets automorphism groups. This designation being indeed more usually used in the Graph Isomorphism context [24]. Anyway, the results that we are going to expose are classical results of group theory concerning the stabilizers and orbits, and then given without proofs. 
Proposition 1. Let $(G, \cdot)$ be a group, and $\phi: G \times \mathbb{F}_{q}[\mathbf{x}]^{u} \rightarrow \mathbb{F}_{q}[\mathbf{x}]^{u}$ be an action of $G$ on $\mathbb{F}_{q}[\mathbf{x}]^{u}$. If there exists $g \in G$, such that $\mathbf{b}=\phi(g, \mathbf{a})$, then $S_{(G, \phi)}(\mathbf{a}, \mathbf{b})$ is a left (resp. right) coset - in $G$ - of the automorphism group $A \operatorname{At}_{(G, \phi)}(\mathbf{a})$ (resp. $\left.\operatorname{Aut}_{(G, \phi)}(\mathbf{b})\right)$. That is:

$$
\left\{\begin{array}{l}
S_{(G, \phi)}(\mathbf{a}, \mathbf{b})=\left\{g \cdot h: h \in A u t_{(G, \phi)}(\mathbf{a})\right\}=g \cdot A u t_{(G, \phi)}(\mathbf{a}), \\
S_{(G, \phi)}(\mathbf{a}, \mathbf{b})=\left\{h \cdot g: h \in A u t_{(G, \phi)}(\mathbf{b})\right\}=A u t_{(G, \phi)}(\mathbf{b}) \cdot g .
\end{array}\right.
$$

Moreover, the automorphism groups $A u t_{(G, \phi)}(\mathbf{a})$ and $A u t_{(G, \phi)}(\mathbf{b})$ are conjugate, i.e. $\operatorname{Aut}_{(G, \phi)}(\mathbf{b})=g \cdot \operatorname{Aut}_{(G, \phi)}(\mathbf{a}) \cdot g^{-1}$, and we have:

$$
\left|S_{(G, \phi)}(\mathbf{a}, \mathbf{b})\right|=\left|A u t_{(G, \phi)}(\mathbf{b})\right|=\left|A u t_{(G, \phi)}(\mathbf{a})\right| .
$$

\subsection{A Generic Upper Bound on the Complexity of "IP-Like" Problems}

Using the Polynomial Equivalence problem previously defined, we give in this part a general upper bound on the theoretical complexity of "IP-like" problems. To do so, Let us fix a group $(G, \cdot)$ acting on $\mathbb{F}_{q}[\mathbf{x}]^{u}$ through a map noted $\phi$.

For simplicity, we suppose here that $G$ is included in a finite set $\mathcal{E}$. We also suppose that the uniform distribution of the elements of $\mathcal{E}$ can be simulated in polynomial-time. These assumptions allows to facilitate the proofs, and are additionally well adapted to "IP-like" problems. Indeed, $A G L_{n}\left(\mathbb{F}_{q}\right) \subset \mathcal{M}_{n, n}\left(\mathbb{F}_{q}\right) \times$ $\mathbb{F}_{q}^{n}, G L_{n}\left(\mathbb{F}_{q}\right) \times G L_{u}\left(\mathbb{F}_{q}\right) \subset \mathcal{M}_{n, n}\left(\mathbb{F}_{q}\right) \times \mathcal{M}_{u, u}\left(\mathbb{F}_{q}\right), A G L_{n}\left(\mathbb{F}_{q}\right) \times A G L_{u}\left(\mathbb{F}_{q}\right) \subset$ $\mathcal{M}_{n, n}\left(\mathbb{F}_{q}\right) \times \mathbb{F}_{q}^{n} \times \mathcal{M}_{u, u}\left(\mathbb{F}_{q}\right) \times \mathbb{F}_{q}^{u}$. To obtain our upper bound, we introduce:

Definition 5. An interactive proof for a language $L$ (i.e. a subset of $\{0,1\}^{*}$ ) is a two party protocol between a verifier $\mathcal{V}$ and a prover $\mathcal{P}$. At the end of the protocol, the verifier has to accept or reject a given input such that the following conditions hold:

Efficiency. The verifier strategy is a probabilistic polynomial time procedure.

Completeness. For all $x \in L, \operatorname{Pr}[(\mathcal{V}, \mathcal{P})(x)$ accepts $]=1$.

Soundness. For all $x \notin L$, and for any prover $\mathcal{P}^{*}, \operatorname{Pr}\left[\left(\mathcal{V}, \mathcal{P}^{*}\right)(x)\right.$ accepts $] \leq \frac{1}{2}$. The probabilities are taken over the random choices of the verifier.

Let us analyse the following two party protocol:

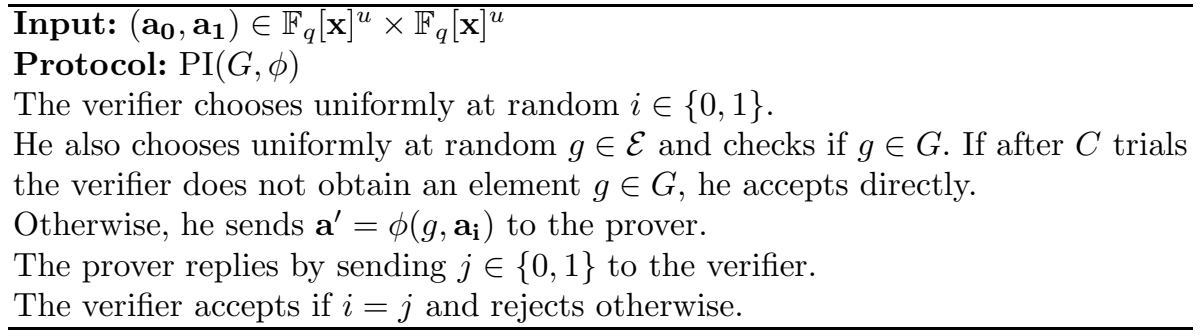

Efficiency. The efficiency of this protocol depends on the cost of computing $\phi\left(g, \mathbf{a}_{\mathbf{i}}\right)$, for all $g \in G$, and of the number of trials $C$. 
Completeness. If $\mathbf{a}_{\mathbf{0}} \not_{(G, \phi)} \mathbf{a}_{\mathbf{1}}$, then a prover can always check if $\mathbf{a}^{\prime} \equiv_{(G, \phi)} \mathbf{a}_{\mathbf{0}}$ or $\mathbf{a}^{\prime} \equiv_{(G, \phi)} \mathbf{a}_{\mathbf{1}}$. In this situation, the verifier accepts with probability one.

Soundness. If $\mathbf{a}_{\mathbf{0}} \equiv_{(G, \phi)} \mathbf{a}_{\mathbf{1}}$, then by transitivity $\mathbf{a}^{\prime} \equiv_{(G, \phi)} \underline{a_{1}}$ and $\mathbf{a}^{\prime} \equiv_{(G, \phi)} \mathbf{a}_{\mathbf{0}}$. In such a case, we will show that $\mathbf{a}^{\prime}=\phi\left(g, \mathbf{a}_{\mathbf{i}}\right)$ yields no information about the bit $i$ chosen by the prover. Let then $\psi$ be a random variable uniformly distributed over $\{0,1\}$, and $\Sigma$ be a random variable uniformly distributed over $G$.

Lemma 2. Let $\mathbf{a}_{\mathbf{0}}, \mathbf{a}_{\mathbf{1}}, \mathbf{a}^{\prime} \in \mathbb{F}_{q}[\mathbf{x}]^{u}$. If $\mathbf{a}_{\mathbf{0}} \equiv_{(G, \phi)} \mathbf{a}_{\mathbf{1}}$ and $\mathbf{a}^{\prime} \equiv_{(G, \phi)} \mathbf{a}_{\mathbf{0}}$, then:

$$
\operatorname{Pr}\left[\psi=0 \mid \mathbf{a}_{\psi}(\mathbf{x} \Sigma)=\mathbf{a}^{\prime}\right]=\operatorname{Pr}\left[\psi=1 \mid \mathbf{a}_{\psi}(\mathbf{x} \Sigma)=\mathbf{a}^{\prime}\right]=\frac{1}{2} .
$$

Proof. We have $\operatorname{Pr}\left[\phi\left(\Sigma, \mathbf{a}_{\psi}\right)=\mathbf{a}^{\prime} \mid \psi=0\right]=\operatorname{Pr}\left[\phi\left(\Sigma, \mathbf{a}_{\mathbf{0}}\right)=\mathbf{a}^{\prime}\right]=\operatorname{Pr}[\Sigma \in$ $\left.S_{(G, \phi)}\left(\mathbf{a}_{\mathbf{0}}, \mathbf{a}^{\prime}\right)\right]$. Moreover, according to Proposition 1:

$$
\left|S_{(G, \phi)}\left(\mathbf{a}_{\mathbf{0}}, \mathbf{a}^{\prime}\right)\right|=\left|A u t_{(G, \phi)}\left(\mathbf{a}^{\prime}\right)\right|=\left|S_{(G, \phi)}\left(\mathbf{a}_{1}, \mathbf{a}^{\prime}\right)\right| .
$$

Therefore, $\operatorname{Pr}\left[\phi\left(\Sigma, \mathbf{a}_{\mathbf{0}}\right)=\mathbf{a}^{\prime}\right]=\operatorname{Pr}\left[\mathbf{a}_{\mathbf{1}}(\mathbf{x} \Sigma)=\mathbf{a}^{\prime}\right]$, and thus:

$$
\operatorname{Pr}\left[\phi\left(\Sigma, \mathbf{a}_{\psi}\right)=\mathbf{a}^{\prime} \mid \psi=0\right]=\operatorname{Pr}\left[\phi\left(\Sigma, \mathbf{a}_{\psi}\right)=\mathbf{a}^{\prime} \mid \psi=1\right] .
$$

According to the Bayes formula:

$$
\begin{aligned}
\left.\operatorname{Pr} \psi=0 \mid \phi\left(\Sigma, \mathbf{a}_{\psi}\right)=\mathbf{a}^{\prime}\right] & =\frac{\operatorname{Pr}[\psi=0] \operatorname{Pr}\left[\phi\left(\Sigma, \mathbf{a}_{\psi}\right)=\mathbf{a}^{\prime} \mid \psi=0\right]}{\operatorname{Pr}\left[\phi\left(\Sigma, \mathbf{a}_{\psi}\right)=\mathbf{a}^{\prime}\right]} \\
& =\frac{\operatorname{Pr}[\psi=1] \operatorname{Pr}\left[\phi\left(\Sigma, \mathbf{a}_{\psi}\right)=\mathbf{a}^{\prime} \mid \psi=1\right]}{\operatorname{Pr}\left[\phi\left(\Sigma, \mathbf{a}_{\psi}\right)=\mathbf{a}^{\prime}\right]} \\
& =\operatorname{Pr}\left[\psi=1 \mid \phi\left(\Sigma, \mathbf{a}_{\psi}\right)=\mathbf{a}^{\prime}\right] .
\end{aligned}
$$

Finally:

$$
\begin{aligned}
\operatorname{Pr}\left[\psi=0 \mid \phi\left(\Sigma, \mathbf{a}_{\psi}\right)=\mathbf{a}^{\prime}\right] & =\frac{\operatorname{Pr}[\psi=0] \operatorname{Pr}\left[\phi\left(\Sigma, \mathbf{a}_{\psi}\right)=\mathbf{a}^{\prime} \mid \psi=0\right]}{\operatorname{Pr}\left[\phi\left(\Sigma, \mathbf{a}_{\psi}\right)=\mathbf{a}^{\prime}\right]} \\
& =\frac{\operatorname{Pr}[\psi=1] \operatorname{Pr}\left[\phi\left(\Sigma, \mathbf{a}_{0}\right)=\mathbf{a}^{\prime}\right]}{\operatorname{Pr}\left[\phi\left(\Sigma, \mathbf{a}_{\psi}\right)=\mathbf{a}^{\prime}\right]} \\
& =\frac{\operatorname{Pr}[\psi=1] \operatorname{Pr}\left[\Sigma \in S_{(G, \psi)}\left(\mathbf{a}^{\prime}, \mathbf{a}_{0}\right)\right]}{\operatorname{Pr}\left[\Sigma \in S_{(G, \phi)}\left(\mathbf{a}_{\psi}, \mathbf{a}^{\prime}\right)\right]}=\frac{1}{2} .
\end{aligned}
$$

It follows that no prover - no matter what its strategy is - can guess $i$ with probability greater than $\frac{1}{2}$. Finally, using a classical result of R. B. Boppana, J. Hastad, and S. Zachos [7], we get that:

Corollary 1. If the polynomial hierarchy does not collapse then IP, 2PLE, and IP1S are not NP-Hard.

Proof. We sketch the proof for IP1S. Note that for all $g \in A G L_{n}\left(\mathbb{F}_{q}\right)$, one can compute $\phi_{\mathrm{IP} 1 \mathrm{~S}}\left(g, \mathbf{a}^{\prime}\right)$ in polynomial-time. Let $\mathrm{L}_{\mathrm{IP}}$ be the language associated to IP1S (i.e. the set of instances of IP admitting a solution). We study now the number of trials in $\operatorname{PI}\left(A G L_{n}\left(\mathbb{F}_{q}\right), \phi_{\mathrm{IP} 1 \mathrm{~S}}\right)$. Recall that more than $1 / 4$ of the matrices of $\mathcal{M}_{n, n}\left(\mathbb{F}_{q}\right)$ are invertible. Therefore for IP1S, we have $G=A G L_{n}\left(\mathbb{F}_{q}\right)$, 
$\mathcal{E}=\mathcal{M}_{n, n}\left(\mathbb{F}_{q}\right) \times \mathbb{F}_{q}^{n}$, and $\operatorname{Pr}[g \in G \mid g \in \mathcal{E}] \geq \frac{1}{4}$. By setting $C=10$, we get that no prover can guess $i$ with probability greater than

$$
\frac{1}{2}+\left(\frac{3}{4}\right)^{10}<\frac{1}{2}+\frac{1}{16}=\frac{9}{16}
$$

where $\left(\frac{3}{4}\right)^{10}<\frac{1}{16}$ is the probability of not obtaining an element of $A G L_{n}\left(\mathbb{F}_{q}\right)$ after ten trials. By repeating the protocol two times, we obtain that no prover

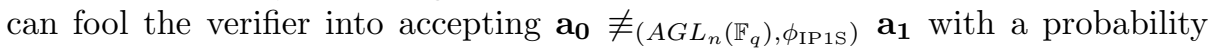
greater than $\left(\frac{9}{16}\right)^{2}<\frac{1}{2}$. The protocol $\mathrm{PI}\left(A G L_{n}\left(\mathbb{F}_{q}\right), \phi_{\mathrm{IP} 1 \mathrm{~S}}\right)$ is then an interactive proof for the complementary language of $\mathrm{L}_{\mathrm{IP} 1 \mathrm{~S}}$ (i.e. $\{0,1\}^{*} \backslash \mathrm{L}_{\mathrm{IP} 1 \mathrm{~S}}$ ), where at most 4 messages are exchanged between the verifier and the prover. We do not detail the proof, but one can easily check that the same result holds for $\operatorname{PI}\left(A G L_{n}\left(\mathbb{F}_{q}\right) \times A G L_{u}\left(\mathbb{F}_{q}\right), \phi_{\mathrm{IP}}\right)$ and $\mathrm{PI}\left(G L_{n}\left(\mathbb{F}_{q}\right) \times G L_{u}\left(\mathbb{F}_{q}\right), \phi_{2 \mathrm{PLE}}\right)$.

The corollary then follows from a result of [7], stating that if the complementary of a language admits a constant round interactive protocol, then this language can not be NP-Complete, unless the polynomial hierarchy collapses.

The new formalism introduced in this part allows to upper bound the theoretical hardness of IP, 2PLE, and IP1S. More generally, it provides a new insight on the complexity of "IP-like" problems. The previous corollary can be indeed easily adapted to any instance of the Polynomial Equivalence problem. An "IP-like" problem is then intrinsically not NP-Hard. Furthermore, we believe that our formalism is of independent interest. It indeed procures a general framework for studying "IP-like" problems. However, this is out of the scope of this paper. We investigate now another aspect of these problems.

\section{An Algorithm for Solving 2PLE}

We study here the practical hardness of a particular Polynomial Equivalence problem, namely 2PLE. Precisely, we present a new algorithm for solving this problem. We emphasize that - as explained in 1.1 - it is usually sufficient to consider this problem rather than its affine variant IP. Besides, any algorithm solving 2PLE can be transformed into an algorithm solving IP [11,12].

\subsection{A First Attempt: Evaluation and Linearization}

Instead of directly describing the details of our method, we present the different steps that yielded to this algorithm. Anyway, most of the intermediate results that we are going to present will be used in our final algorithm, but differently. Our earliest idea for solving $2 \mathrm{PLE}$ was based on the following remark. If $\mathbf{b}(\mathbf{x})=$ $\mathbf{a}(\mathbf{x} S) U$, for $(S, U) \in G L_{n}\left(\mathbb{F}_{q}\right) \times G L_{u}\left(\mathbb{F}_{q}\right)$, then:

$$
\mathbf{b}(\mathbf{p}) U^{-1}=\mathbf{a}(\mathbf{p} S) \text {, for all } \mathbf{p} \in \mathbb{F}_{q}^{n} .
$$

We hence obtain, for each $\mathbf{p} \in \mathbb{F}_{q}^{n}, u$ non-linear equations in the $n^{2}+u^{2}$ components of the matrices $S$ and $U^{-1}$. We point out that the coefficients of $U^{-1}$ only 
appear linearly in these equations. This is the advantage of considering the inverse of $U$ rather than simply $U$ in (1). The number of equations obtained is then significantly bigger than the number of unknowns. In this situation, one can simply use a linearization method (i.e. associating a new variable to each monomial) for solving the algebraic system. Unfortunately, our experiments rapidly revealed that the equations generated in this way are not all linearly independent. Besides, it also appeared that the number of unknowns is significantly bigger than the number of linearly independent equations. The use of a linearization method is then clearly no longer relevant. Let us explain this phenomenon.

Lemma 3. Let $\mathbf{y}=\left(y_{1,1}, \ldots, y_{1, n}, \ldots, y_{n, 1}, \ldots, y_{n, n}\right)$, and $\mathbf{z}=\left(z_{1,1}, \ldots, z_{1, u}, \ldots\right.$ $\left., z_{u, 1}, \ldots, z_{u, u}\right)$. For each $i, 1 \leq i \leq u$, there exists a subset $S_{i} \subseteq \mathbb{F}_{q}^{n}$ and polynomials $p_{\alpha, i} \in \mathbb{F}_{q}[\mathbf{y}, \mathbf{z}]$, such that the following equality holds:

$$
\left(\mathbf{b}(\mathbf{x}) U^{-1}-\mathbf{a}(\mathbf{x} S)\right)_{i}=\sum_{\alpha \in S_{i}} p_{\alpha, i}\left(S, U^{-1}\right) \mathbf{x}^{\alpha},
$$

$p_{\alpha, i}\left(S, U^{-1}\right)$ being the evaluation of $p_{\alpha, i}$ on $S=\left\{s_{i, j}\right\}_{1 \leq i, j \leq n}, U^{-1}=\left\{u_{i, j}^{\prime}\right\}_{1 \leq i, j \leq u}$.

Proof. The polynomial $\left(\mathbf{b}(\mathbf{x}) U^{-1}-\mathbf{a}(\mathbf{x} S)\right)_{i}$ can be regarded as an element of:

$$
\mathbb{F}_{q}\left[s_{1,1}, \ldots, s_{1, n}, \ldots, s_{n, 1}, \ldots, s_{n, n}, u_{1,1}^{\prime}, \ldots, u_{1, u}^{\prime}, \ldots, u_{u, u}^{\prime}, \ldots, u_{u, u}^{\prime}\right]\left[x_{1}, \ldots, x_{n}\right],
$$

i.e. a polynomial with unknowns $x_{1}, \ldots, x_{n}$ and whose coefficients are polynomials in the components of $S$ and $U^{-1}$. In this setting, the polynomials $p_{\alpha, i}$ exactly correspond to the coefficients of the monomials (in $x_{1}, \ldots, x_{n}$ ) occurring in $\left(\mathbf{b}(\mathbf{x}) U^{-1}-\mathbf{a}(\mathbf{x} S)\right)_{i}$. Lastly $S_{i}=\left\{\alpha \in \mathbb{F}_{q}^{n}: p_{\alpha, i} \neq 0\right\}$.

The cost of generating the polynomials $p_{\alpha, i}$ is proportional to the number of monomials occurring in $\left(\mathbf{b}(\mathbf{x}) U^{-1}-\mathbf{a}(\mathbf{x} S)\right)_{i}$ viewed as a polynomial of (3), i.e. $O\left(n^{2 D}\right)$. Note also that each $p_{\alpha, i}$ is by construction the sum of a polynomial in $\mathbf{y}$, plus a linear polynomial in $\mathbf{z}$. Furthermore, the maximal total degree reached by a monomial in the variables $\mathbf{y}$ is equal to $D$.

From (2), we obtain that for all $i, 1 \leq i \leq u$ :

$$
\left(\mathbf{b}(\mathbf{p}) U^{-1}-\mathbf{a}(\mathbf{p} S)\right)_{i}=\sum_{\alpha \in S_{i}} p_{\alpha, i}\left(S, U^{-1}\right) p_{1}^{\alpha_{1}} \cdots p_{n}^{\alpha_{n}}, \text { for all } \mathbf{p}=\left(p_{1}, \ldots, p_{n}\right) \in \mathbb{F}_{q}^{n} .
$$

It follows that, for all $\mathbf{p} \in \mathbb{F}_{q}^{n}$, the equations procured by (1) are linear combinations of the $p_{\alpha, i}\left(S, U^{-1}\right)$. The number of polynomials $p_{\alpha, i}$ is limited by the number of monomials occuring in $\left(\mathbf{b}(\mathbf{p}) U^{-1}-\mathbf{a}(\mathbf{p} S)\right)_{i}$. Thus, $u \cdot \mathrm{C}_{n+D}^{D}$ bounds from above the number of linearly independent equations provided by linearizing (1). On the other hand, the number of unknowns in the linearized system is equal to the number of monomials in the variables $\mathbf{y}$ of degree smaller than $D$, plus the $u^{2}$ variables corresponding to $\mathbf{z}$. Using a rough bound, the linearization method yields a linear system of at most $O\left(u \cdot n^{D}\right)$ linearly independent equations with $O\left(u \cdot n^{2 D}\right)$ unknowns. 


\subsection{The 2PLE Algorithm}

The linearization can thus not be employed for solving efficiently 2PLE. However, Gröbner basis procures another method for solving the algebraic system given by (1). From a practical point of view, this approach is quite promising. Indeed, the system obtained by evaluating $\mathbf{b}(\mathbf{x}) U^{-1}=\mathbf{a}(\mathbf{x} S)$ on several vectors is overdetermined. Nevertheless, all the equations derived from $\mathbf{b}(\mathbf{p}) U^{-1}=\mathbf{a}(\mathbf{p} S)$ are according to (2) linear combinations the polynomials $p_{\alpha, i}$. It is hence sufficient to only consider the system formed by these equations. Formally:

Proposition 2. Let $\mathcal{I}=\left\langle p_{\alpha, i}\right.$ : for all $i, 1 \leq i \leq u$, and for all $\left.\alpha \in S_{i}\right\rangle \subset$ $\mathbb{F}_{q}[\mathbf{y}, \mathbf{z}]$ be the ideal generated by the polynomials $p_{\alpha, i}$ defined as in Lemma 3 , and $V(\mathcal{I})$ be the following variety:

$$
\begin{gathered}
V(\mathcal{I})=\left\{\mathbf{s} \in \mathbb{F}_{q}^{n^{2}+u^{2}}: p_{\alpha, i}(\mathbf{s})=0, \text { for all } i, 1 \leq i \leq u, \text { and for all } \alpha \in S_{i}\right\} . \\
\text { If } \mathbf{b}(\mathbf{x})=\mathbf{a}(\mathbf{x} S) U \text {, for some }(S, U) \in G L_{n}\left(\mathbb{F}_{q}\right) \times G L_{u}\left(\mathbb{F}_{q}\right), \text { then: } \\
\left(\phi_{1}(S), \phi_{2}\left(U^{-1}\right)\right) \in V(\mathcal{I}),
\end{gathered}
$$

with:

$$
\begin{aligned}
& \phi_{1}: \mathcal{M}_{n, n}\left(\mathbb{F}_{q}\right) \rightarrow \mathbb{F}_{q}^{n^{2}}, S=\left\{s_{i, j}\right\}_{1 \leq i, j \leq n} \mapsto\left(s_{1,1}, \ldots, s_{1, n}, \ldots, s_{n, 1}, \ldots, s_{n, n}\right), \text { and } \\
& \phi_{2}: \mathcal{M}_{u, u}\left(\mathbb{F}_{q}\right) \rightarrow \mathbb{F}_{q}^{u^{2}}, U^{-1}=\left\{u_{i, j}^{\prime}\right\}_{1 \leq i, j \leq u} \mapsto\left(u_{1,1}^{\prime}, \ldots, u_{1, u}^{\prime}, \ldots, u_{u, 1}^{\prime}, \ldots, u_{u, u}^{\prime}\right) .
\end{aligned}
$$

Proof. For all, $i, 1 \leq i \leq u$ :

$$
\left(\mathbf{b}(\mathbf{x}) U^{-1}-\mathbf{a}(\mathbf{x} S)\right)_{i}=\sum_{\alpha \in S_{i}} p_{\alpha, i}\left(S, U^{-1}\right) \mathbf{x}^{\alpha}=0 .
$$

Thus, $p_{\alpha, i}\left(S, U^{-1}\right)=0, \forall i, 1 \leq i \leq u$, and $\forall \alpha \in S_{i}$, i.e. $\left(\phi_{1}(S), \phi_{2}\left(U^{-1}\right)\right) \in V(\mathcal{I})$.

In other words, if $\mathbf{b}=\mathbf{a}(\mathbf{x} S) U$, for some $(S, U) \in G L_{n}\left(\mathbb{F}_{q}\right) \times G L_{u}\left(\mathbb{F}_{q}\right)$, then the variety $V(\mathcal{I})$ contains the components of the matrices $S$ and $U^{-1}$. The system associated to $\mathcal{I}$ has $n^{2}+u^{2}$ variables and is of degree $D$. Once again, we recall that the variables of $\mathbf{z}$ only appear linearly in this system. The number of equations of the system is equal to the number of monomials occurring in the polynomials of a, i.e. $O\left(u \cdot \mathrm{C}_{n+D}^{D}\right)$. The system is then overdetermined.

Remark 2. In order to guarantee that $V(\mathcal{I}) \subseteq \mathbb{F}_{q}^{2 n}$, we must generally join the field equations to the initial system. The fields considered in our case can be relatively large, leading then to a significant increase of the system's degree. This can artificially render impracticable the computation of a Gröbner basis. Fortunately, our systems are overdetermined and it is not necessary in practice to include the field equations. In our experiments the elements of $V(\mathcal{I})$ were indeed - without including these equations - all the times in $\mathbb{F}_{q}^{2 n}$. It implies in particular that the hardness of 2PLE is not related to the size of the field. This is an important remark since the current security bound for 2PLE depends on this size. 
The next proposition is fundamental to understand the practical behaviour of our approach. This result permits furthermore to improve the efficiency of our method.

Proposition 3. Let $d$ be a positive integer, and $\mathcal{I}_{d} \subset \mathbb{F}_{q}[\mathbf{y}, \mathbf{z}]$ be the ideal generated by the polynomials $p_{\alpha, i}$ of maximal total degree smaller than $d$. Let also $V\left(\mathcal{I}_{d}\right)$ be the variety associated to $\mathcal{I}_{d}$. If $\mathbf{b}(\mathbf{x})=\mathbf{a}(\mathbf{x} S) U$, for some $(S, U) \in$ $G L_{n}\left(\mathbb{F}_{q}\right) \times G L_{u}\left(\mathbb{F}_{q}\right)$, then:

$$
\left(\phi_{1}(S), \phi_{2}\left(U^{-1}\right)\right) \in V\left(\mathcal{I}_{d}\right), \text { for all } d, 0 \leq d \leq D,
$$

$\phi_{1}$ and $\phi_{2}$ being defined as in proposition 2.

The proof is obviously deduced from the following result:

Lemma 4. Let $(S, U) \in G L_{n}\left(\mathbb{F}_{q}\right) \times G L_{u}\left(\mathbb{F}_{q}\right)$. We have:

$$
\mathbf{b}(\mathbf{x})=\mathbf{a}(\mathbf{x} S) U \Longleftrightarrow \mathbf{b}^{(d)}(\mathbf{x})=\mathbf{a}^{(d)}(\mathbf{x} S) U, \text { for all } d, 0 \leq d \leq D,
$$

$\mathbf{b}^{(d)}$ (resp. $\left.\mathbf{a}^{(d)}\right)$ being the homogeneous components of degree d (i.e. the sum of the terms of total degree $d$ ) of the polynomials of $\mathbf{b}$ (resp. $\mathbf{a}$ ).

The systems associated to $\mathcal{I}_{1}$ and $\mathcal{I}_{0}$ only contain linear equations in the components of $S$ and $U^{-1}$. Indeed, let $\mathbf{0}_{\mathbf{n}}$ be the null vector of $\mathbb{F}_{q}^{n}$, and $A \in$ $\mathcal{M}_{n, u}\left(\mathbb{F}_{q}\right)\left(\right.$ resp. $\left.B \in \mathcal{M}_{n, u}\left(\mathbb{F}_{q}\right)\right)$ be the matrix representation of $\mathbf{a}^{(1)}$ (resp. $\left.\mathbf{b}^{(1)}\right)$, i.e. $\mathbf{x} A=\mathbf{a}^{(1)}(\mathbf{x})\left(\operatorname{resp} . \mathbf{x} B=\mathbf{b}^{(1)}(\mathbf{x})\right)$. According to Lemma 4 :

$\mathbf{b}=\mathbf{a}(\mathbf{x} S) U$, for $(S, U) \in G L_{n}\left(\mathbb{F}_{q}\right) \times G L_{u}\left(\mathbb{F}_{q}\right) \Longrightarrow\left\{\begin{array}{l}\mathbf{b}^{(0)}\left(\mathbf{0}_{\mathbf{n}}\right) U^{-1}=\mathbf{a}^{(0)}\left(\mathbf{0}_{\mathbf{n}}\right), \\ B U^{-1}=S A\end{array}\right.$

That is, we get linear dependencies between the components $S$ and $U^{-1}$. More precisely, we obtain $u(n+1)$ linear equations in the $n^{2}+u^{2}$ components of the matrices solution. Anyway, we can not solve 2PLE just by using these equations. On the other hand, it is not necessary to consider the system formed by all the polynomials $p_{\alpha, i}$. According to Proposition 3, we can actually restrict our attention on $\mathcal{I}_{d_{0}}$, with $d_{0}$ being the smaller integer rendering the system overdetermined. This $d_{0}$ can be defined in function of $\mathbf{a}$. Indeed, $d_{0} \approx \min \left\{d>1: \mathbf{a}^{(d)} \neq \underline{0_{u}}\right\}$. In practice, it is usually sufficient to take $d_{0}=2$. The hardness of an instance of $2 \mathrm{PLE}$ is then related to $d_{0}$ rather than to the maximal total degree $D$ of this instance. It is also an important remark since the maximal degree of an instance is taken into account in the security estimate of 2PLE given by J. Patarin [28, 29]. Our algorithm for solving this problem is as follows:

Input: $(\mathbf{a}, \mathbf{b}) \in \mathbb{F}_{q}[\mathbf{x}]^{u} \times \mathbb{F}_{q}[\mathbf{x}]^{u}$

Let $d_{0}=\min \left\{d>1: \mathbf{a}^{(d)} \neq \mathbf{0}_{\mathbf{u}}\right\}$

Construct the polynomials $p_{\alpha, i}$ of max. total deg. smaller than $d_{0}$

Compute $V\left(\mathcal{I}_{d_{0}}\right)$ using the $\mathrm{F}_{5}$ algorithm

Find an element of $V\left(\mathcal{I}_{d_{0}}\right)$ corresponding to a solution of $2 \mathrm{PLE}$

Return this solution 
The system associated to $\mathcal{I}_{d_{0}}$ is overdetermined by its very construction $\left(u^{2}+n^{2}\right.$ unknowns, and $O\left(u \cdot \mathrm{C}_{n+d_{0}}^{d_{0}}\right)$ equations). The variety $V\left(\mathcal{I}_{d_{0}}\right)$ is then very likely reduced to a solution of $2 \mathrm{PLE}$ (this has been indeed verified in our experiments). The complexity of this algorithm is (theoretically) dominated by the Gröbner basis computation. It is difficult to obtain a complexity bound really reflecting the practical behavior of the $\mathrm{F}_{5}$ algorithm. We therefore carry out now experimental results illustrating the practical efficiency of our approach.

\subsection{Experimental Results}

We present in this part experimental results obtained with our algorithm. Before that, we provide the conditions of our experiments

\section{Generation of the instances}

We have only considered instances $(\mathbf{a}, \mathbf{b})$ of 2PLE admitting a solution. We constructed the instances in the following way:

(1) Choose the polynomials of a

(2) Randomly choose $(S, U) \in G L_{n}\left(\mathbb{F}_{q}\right) \times G L_{u}\left(\mathbb{F}_{q}\right)$

(3) Return $(\mathbf{a}(\mathbf{x}), \mathbf{b}(\mathbf{x})=\mathbf{a}(\mathbf{x} S) U)$

Precisely, we constructed the polynomials of a in two different ways. The first one simply consists in randomly choosing - w.r.t. a given maximal total degree $D$ - the polynomials of a. Precisely, each polynomial is a random linear combination of all the monomials of total degree smaller (or equal) to $D$. Note that we obtain in this way dense polynomials. We shall call random instance, an instance of 2PLE generated in this manner. In the second method, a corresponds to the public key of a $\mathrm{C}^{*}$ scheme [26]. An instance of 2PLE generated in this way will be named $C^{*}$ instance.

\section{Programming Language - Workstation}

The experimental results have been obtained with an Opteron bi-processors 2.4 Ghz, with $8 \mathrm{~Gb}$ of Ram. The systems associated to an instance of 2PLE have been generated using the Magma software[25]. We used our own implementation (in language $\mathrm{C}$ ) of $\mathrm{F}_{5}$ for computing the Gröbner bases. However, for the sake of comparison, we sometimes used the last version of Magma (i.e. 2.12) for obtaining these bases. This version includes an implementation of the $\mathrm{F}_{4}$ algorithm.

\section{Table Notations}

The following notations are used in the tables below:

$-n$, the number of variables,

$-q$, the size of the field,

- deg, the maximal total degree of the considered instance,

- $T_{G e n}$, the time needed to construct the system,

$-T_{F_{5}}$, the time of our algorithm for finding a solution of 2PLE (using the $\mathrm{F}_{5}$ algorithm for computing the Gröbner bases,

$-T$, the total time of our algorithm, i.e. $T=T_{F_{5}}+T_{G e n}$, 
$-T_{F_{4} / \text { Mag }}$, the time of our algorithm for recovering a solution of $2 \mathrm{PLE}$, using Magma v. 2.12 for computing Gröbner bases,

$-q^{n / 2}$ (resp. $q^{n}$ ), the security bound given in $[11,12]$ for instances of deg $=2$ (resp. $\operatorname{deg}>2$ ).

\section{Practical Results - Random Instances}

We present here the results obtained on random instances of 2PLE. We emphasize that this family of instances is the one employed in the authentication and signature schemes based on 2PLE proposed by J. Patarin at Eurocrypt'96 $[28,29]$. He suggested to use $u=n$ in practice. Since our main motivation is to study the security of these schemes, we can restrict our attention on the case $u=n$.

\begin{tabular}{|c|c|c|c|c|c|c|c|}
\hline$n$ & $q$ & $d e g$ & $T_{G e n}$ & $T_{F_{5}}$ & $T_{F_{4} / M a g} / T_{F_{5}}$ & $T$ & $q^{n / 2}$ \\
\hline 8 & $2^{16}$ & 2 & $0.35 \mathrm{~s}$. & $0.14 \mathrm{~s}$. & 6 & $0.49 \mathrm{~s}$. & $2^{64}$ \\
\hline 10 & $2^{16}$ & 2 & $1.66 \mathrm{~s}$. & $0.63 \mathrm{~s}$. & 10 & $2.29 \mathrm{~s}$. & $2^{80}$ \\
\hline 12 & $2^{16}$ & 2 & $7.33 \mathrm{~s}$. & $2.16 \mathrm{~s}$. & 16 & $9.49 \mathrm{~s}$. & $2^{96}$ \\
\hline 15 & $2^{16}$ & 2 & $48.01 \mathrm{~s}$. & $10.9 \mathrm{~s}$. & 23 & $58.91 \mathrm{~s}$. & $2^{120}$ \\
\hline 17 & $2^{16}$ & 2 & $137.21 \mathrm{~s}$. & $27.95 \mathrm{~s}$. & 31 & $195.16 \mathrm{~s}$. & $2^{136}$ \\
\hline 20 & $2^{16}$ & 2 & $569.14 \mathrm{~s}$. & $91.54 \mathrm{~s}$. & 41 & $660.68 \mathrm{~s}$. & $2^{160}$ \\
\hline \hline 10 & 65521 & 2 & $1.21 \mathrm{~s}$. & $0.44 \mathrm{~s}$. & 10 & $1.65 \mathrm{~s}$. & $\approx 2^{80}$ \\
\hline 15 & 65521 & 2 & $35.58 \mathrm{~s}$. & $8.08 \mathrm{~s}$. & 23 & $43.66 \mathrm{~s}$. & $\approx 2^{120}$ \\
\hline 20 & 65521 & 2 & $434.96 \mathrm{~s}$. & $69.96 \mathrm{~s}$. & 41 & $504.92 \mathrm{~s}$. & $\approx 2^{160}$ \\
\hline 23 & 65521 & 2 & $1578.6 \mathrm{~s}$. & $235.92 \mathrm{~s}$. & & $1814 \mathrm{~s}$. & $\approx 2^{184}$ \\
\hline
\end{tabular}

Remark 3. Our implementation of $F_{5}$ is faster than the Gröbner basis algorithm available in Magma 2.12. For $n=20, F_{5}$ is for instance 41 times faster than Magma. To fix ideas, $u=n=8$, and $u=n=16$ were two challenges proposed at Eurocrypt'96 [29]. We obtained exactly the same results as the ones quoted in the previous table for random instances of deg $>2$. On the other hand, the security estimate for these instances is at least equal to $2^{128}(n=8)$. The maximal total degree of the systems is indeed the same as for instances of deg $=2$, i.e. $d_{0}$ is equal to 2 independently of $D$. In other words, increasing the maximal total degree of a random instance will not change its practical hardness. We observe the same behavior for the size of the field, that is increasing $q$ does not really change the hardness of a random instance. This will indeed modify only the cost of the arithmetic operations in the different steps our algorithm.

\section{Interpretation of the Results}

In all these experiments, the varieties computed were reduced to one element, i.e. the components of the matrices solution of 2PLE. Furthermore, we observe in practice that the complexity of our algorithm is dominated by the time required to construct the system, and not by the Gröbner basis computation. This is surprising, but it clearly highlights that the systems considered here can be easily solved in practice. The generation of the systems being polynomial, we then conclude experimentally that our algorithm solves random instances of 
2PLE in polynomial-time. This conclusion is supported by the fact that in all these experiments, the matrices generated by $\mathrm{F}_{5}$ (see the Appendix) were of size at most equal to $n^{3}$. Experimentally, we deduce a complexity of $\left(n^{3}\right)^{3}=n^{9}$ for our algorithm on random instances of $2 \mathrm{PLE}$.

\section{Practical Results - C* Instances}

We now present the results obtained on $\mathrm{C}^{*}$ instances $(\mathbf{a}, \mathbf{b})$ of degree $D$. We highlight that these instances are used in the traitor tracing scheme described in [5]. In this context, we also have $u=n$. The polynomials of a correspond to the public-key of a $\mathrm{C}^{*}$ scheme [26]. Precisely, these polynomials are the "multivariate representation" of a univariate monomial (see [5] for details concerning the generation of this multivariate representation). The univariate monomial has the following shape: $m^{1+q^{\theta_{1}}+q^{\theta_{2}}+\cdots+q^{\theta-1}}$, with $\theta_{1}, \theta_{2}, \cdots, \theta_{D-1} \in \mathbb{N}^{*}$.

\begin{tabular}{|c|c|c|c|c|c|c|c|}
\hline$n$ & $q$ & $d e g$ & $T_{G e n}$ & $T_{F_{5}}$ & $T_{F_{4} / M a g} / T_{F_{5}}$ & $T$ & $q^{n}$ \\
\hline 5 & $2^{16}$ & 4 & $0.2 \mathrm{~s}$. & $0.13 \mathrm{~s}$. & 45 & $0.33 \mathrm{~s}$. & $2^{80}$ \\
\hline 6 & $2^{16}$ & 4 & $0.7 \mathrm{~s}$. & $1.03 \mathrm{~s}$. & 64 & $1.73 \mathrm{~s}$. & $2^{96}$ \\
\hline 7 & $2^{16}$ & 4 & $1.5 \mathrm{~s}$. & $6.15 \mathrm{~s}$. & 90 & $7.65 \mathrm{~s}$. & $2^{112}$ \\
\hline 8 & $2^{16}$ & 4 & $3.88 \mathrm{~s}$. & $54.34 \mathrm{~s}$. & 112 & $58.22 \mathrm{~s}$. & $2^{128}$ \\
\hline 9 & $2^{16}$ & 4 & $5.43 \mathrm{~s}$. & $79.85 \mathrm{~s}$. & 145 & $85.28 \mathrm{~s}$. & $2^{144}$ \\
\hline 10 & $2^{16}$ & 4 & $12.9 \mathrm{~s}$. & $532.33 \mathrm{~s}$. & 170 & $545.23 \mathrm{~s}$. & $2^{160}$ \\
\hline
\end{tabular}

Remark 4. $n=5$, and deg $=4$ is the first challenge proposed at Asiacrypt'03 [5]. Similarly to random instances, we observed that the size of the field does not really change the practical hardness of the $C^{*}$ instances. We can conclude that it is a general behaviour of $2 P L E$ instances.

\section{Interpretation of the Results and Future Work}

Our algorithm is no longer polynomial for $\mathrm{C}^{*}$ instances. The systems obtained for these instances are indeed harder to solve than the random ones. We believe that it is due to the fact that the systems are here sparser. The equality $\mathbf{b}\left(\mathbf{0}_{\mathbf{n}}\right)=\mathbf{a}\left(\mathbf{0}_{\mathbf{n}}\right) U$ does not provide any information $\left(\mathbf{b}\left(\mathbf{0}_{\mathbf{n}}\right)=\mathbf{a}\left(\mathbf{0}_{\mathbf{n}}\right)=\mathbf{0}_{\mathbf{n}}\right.$ in the $\mathrm{C}^{*}$ case). It is not clear yet but it seems that $\mathrm{C}^{*}$ instances with $n=19$ (the second challenge proposed in [5]), can not be solved with our approach.

More generally, we think that $d_{0}=\min \left\{d \geq 0: \mathbf{a}^{(d)} \neq \mathbf{0}_{\mathbf{u}}\right\}$ provides a relevant measure of the practical hardness of $2 \mathrm{PLE}$ instances. It seems actually that this practical difficulty increases in function of $d_{0}$. Indeed, for random instances of $2 \mathrm{PLE}, d_{0}=0$ and our algorithm solves $2 \mathrm{PLE}$ efficiently. For $\mathrm{C}^{*}$ instances, $d_{\min }=1$ and there is a change of complexity class. We also checked that the practical complexity increases for homogeneous instances of degree 2 , i.e. $d_{0}=2$. To summarize, for $d_{0}=0$ it is relatively clear that our algorithm solves 2PLE efficiently (likely in polynomial-time). For $d_{0} \geq 1$, we conjecture that our algorithm is subexponential in $n$, and will depend on $d_{0}$. This anyway needs further investigations. It is an open problem to precisely determine, as a function of $d_{0}$, the asymptotic complexity of our algorithm. It could be possible that techniques presented in $[3,4]$ provide an answer. 


\section{Acknowledgements}

We thank Françoise Levy-dit-Vehel and anonymous referees for numerous comments which improved the presentation of the results.

\section{References}

1. W.W. Adams and P. Loustaunau. An Introduction to Gröbner Bases. Graduate Studies in Mathematics, Vol. 3, AMS, 1994.

2. G. Ars, J.-C. Faugère, H. Imai, M. Kawazoe, and M. Sugita. Comparison Between $X L$ and Gröbner Basis Algorithms. Advances in Cryptology - ASIACRYPT 2004, Lecture Notes in Computer Science, vol. 3329, pp. 338-353, 2004.

3. M. Bardet, J-C. Faugère, B. Salvy and B-Y. Yang. Asymptotic Behaviour of the Degree of Regularity of Semi-Regular Polynomial Systems. In MEGA 2005, Eighth International Symposium on Effective Methods in Algebraic Geometry, 15 pages, 2005.

4. M. Bardet, J-C. Faugère, and B. Salvy. On the Complexity of Gröbner Basis Computation of Semi-Regular Overdetermined Algebraic Equations. In Proc. of International Conference on Polynomial System Solving (ICPSS), pp. 71-75, 2004.

5. O. Billet, and H. Gilbert. A Traceable Block Cipher. Advances in Cryptology ASIACRYPT 2003, Lecture Notes in Computer Science, vol. 2894, Springer-Verlag, pp. 331-346, 2003.

6. A. Biryukov, C. De Cannière, A. Braeken, and B. Preneel. A Toolbox for Cryptanalysis: Linear and Affine Equivalence Algorithms. Advances in Cryptology - EUROCRYPT 2003, Lecture Notes in Computer Science, vol. 2656, Springer-Verlag, pp. 33-50, 2003.

7. R. B. Boppana, J. Hastad, and S. Zachos. Does co-NP Have Short Interactive Proofs? Information Processing Letters, 25(2), pp. 127-132, 1987.

8. B. Buchberger. Gröbner Bases : an Algorithmic Method in Polynomial Ideal Theory. Recent trends in multidimensional systems theory. Reider ed. Bose, 1985.

9. B. Buchberger, G.-E. Collins, and R. Loos. Computer Algebra Symbolic and Algebraic Computation. Springer-Verlag, second edition, 1982.

10. N. Courtois. La sécurité des primitives cryptographiques basées sur des problèmes algébriques multivariables: MQ, IP, MinRank, HFE. Ph.D. Thesis, Paris, 2001.

11. N. Courtois, L. Goubin, and J. Patarin. Improved Algorithms for Isomorphism of Polynomials. Advances in Cryptology - EUROCRYPT 1998, Lecture Notes in Computer Science, vol. 1403, Springer-Verlag, pp. 84-200, 1998.

12. N. Courtois, L. Goubin, and J. Patarin. Improved Algorithms for Isomorphism of Polynomials - Extended Version. Available from http://www.minrank.org.

13. N. Courtois, L. Goubin, and J. Patarin. SFLASH, a Fast Asymmetric Signature Scheme for low-cost Smartcards - Primitive Specification and Supporting Documentation. Available at http://www.minrank.org/sflash-b-v2.pdf.

14. N. Courtois, A. Klimov, J. Patarin, and A. Shamir. Efficient Algorithms for Solving Overdefined Systems of Multivariate Polynomial Equations. Advances in Cryptology - EUROCRYPT 2000, Lecture Notes in Computer Science, vol. 1807, SpringerVerlag, pp. 392-407, 2000.

15. D. A. Cox, J.B. Little and, D. O'Shea. Ideals, Varieties, and Algorithms: an Introduction to Computational Algebraic Geometry and Commutative Algebra. Undergraduate Texts in Mathematics. Springer-Verlag. New York, 1992. 
16. J.-C. Faugère. A New Efficient Algorithm for Computing Gröbner Bases $\left(F_{4}\right)$. Journal of Pure and Applied Algebra, 139(1-3), pp. 61-88, June 1999.

17. J.-C. Faugère. A New Efficient Algorithm for Computing Gröbner Basis without Reduction to Zero: $\mathrm{F}_{5}$. Proceedings of ISSAC, pp. 75-83. ACM press, July 2002.

18. J.-C. Faugère, and A. Joux. Algebraic Cryptanalysis of Hidden Field Equation (HFE) Cryptosystems using Gröbner bases. Advances in Cryptology - CRYPTO 2003, Lecture Notes in Computer Science, vol. 2729, Springer-Verlag, pp. 44-60, 2003.

19. J. C. Faugère, P. Gianni, D. Lazard, and T. Mora. Efficient Computation of ZeroDimensional Gröbner Bases by Change of Ordering. Journal of Symbolic Computation, 16(4), pp. 329-344, 1993.

20. P. Felke On certain Families of HFE-type Cryptosystems. Proceedings of WCC'05, International Workshop on Coding and Cryptography, March 2005.

21. S. Fortin. The Graph Isomorphism problem. Technical Report 96-20, University of Alberta, 1996.

22. M. R. Garey, and D. B. Johnson. Computers and Intractability. A Guide to the Theory of NP-Completeness. W. H. Freeman, 1979.

23. W. Geiselmann, R. Steinwandt, and T. Beth. Attacking the Affine Parts of SFLASH. Cryptography and Coding, 8th IMA International Conference, vol. 2260, Springer-Verlag, pp. 355-359, 2001.

24. M. Hoffman. Group-theoretic algorithms and Graph Isomorphism. Lecture Notes in Computer Science, vol. 136, Springer-Verlag, 1982.

25. http://magma.maths.usyd.edu.au/magma/

26. T. Matsumoto, and H. Imai. Public Quadratic Polynomial-tuples for efficient signature-verification and message-encryption. Advances in Cryptology - EUROCRYPT 1988, Lecture Notes in Computer Science, vol. 330, Springer-Verlag, pp. 419-453, 1988.

27. https://www.cosic.esat.kuleuven.be/nessie/deliverables/decision-final.pdf.

28. J. Patarin. Hidden Fields Equations (HFE) and Isomorphisms of Polynomials (IP): two new families of Asymmetric Algorithms. Advances in Cryptology - EUROCRYPT 1996, Lecture Notes in Computer Science, vol. 1070, Springer-Verlag, pp. 33-48, 1996.

29. J. Patarin. Hidden Fields Equations (HFE) and Isomorphisms of Polynomials (IP): two new families of Asymmetric Algorithms - Extended Version. Available from http://www.minrank.org/hfe/.

30. J. Patarin. Cryptanalysis of the Matsumoto and Imai Public Key Scheme of Eurocrypt'88. Advances in Cryptology - CRYPTO 1995, Lecture Notes in Computer Science, Springer-Verlag, vol. 963, pp. 248-261, 1995. 